\title{
Application of computational fluid dynamics to study the influence of turbulence models in the behavior of the cyclonic separators
}

\begin{abstract}
This paper applies computational fluid dynamics to study the influence of turbulence models in the behavior of cyclonic separators, we used different turbulence models to model the behavior of single phase air into the cyclone separator, between them, Standard k-epsilon, RNG k-epsilon, Realizable k-epsilon and Spalart Allamara model, employment numerically with FLUENT ${ }^{\circledR}$ code in its version 6.3 , using the finite volume method, we compared the tangential velocity profiles obtained numerically with experimental data, the finite volume method applied to fluid dynamics problems is a useful and important tool for the conceptualization of the phenomenon to be studied, establishing a relationship between the approximation schemes used and the physical effects involved in transport phenomena analyze.
\end{abstract}

Keywords, cyclones, computational fluid dynamics, turbulence
Volume 2 Issue 2 - 2017

\author{
Héctor Zambrano Meza, Linda Margarita \\ Medina \\ Department of Physics and Mathematics, Monterrey Institute of \\ Technology and Higher Education, México
}

\begin{abstract}
Correspondence: Héctor Zambrano Meza, Department of Physics and Mathematics, Monterrey Institute of Technology and Higher Education, México,
\end{abstract}

Email hectorj.zambranom@gmail.com

Received: March 01, 2017| Published: March 22, 2017
Abbreviations, $a$, velocity of sound; $\rho$, density; $P k$, represents the generation of turbulent kinetic energy due to the velocity gradient; $G k$, is the generation of turbulent kinetic energy due to floating forces; $\mu_{t}$, is the turbulent viscosity; $\mathrm{C}_{1 \varepsilon}$ y $\mathrm{C}_{2 \varepsilon}$, constants; $\sigma_{k}, \sigma_{\varepsilon}$, are the turbulent prandtl numbers for the k-epsilon equations respectively; $Y_{M}$, represents the contribution of fluctuations of the expansion in the compressible turbulence; $\rho$, is the density; $S_{h}$, are the source terms which include contributions due only to the forces of the body; $M_{t}$, turbulent mach number; $\overline{\Omega_{i j}}$, is the rotation tensor seen from the reference point of the angular velocity $\left(w_{\kappa}\right)$

\section{Introduction}

Cyclonic separators are currently widely applied and accepted at industrial level due to their simplicity of construction, low operating costs, the absence of moving parts, low energy requirements ${ }^{1}$ and to be adaptable to A wide range of operation, its use covers industries such as cement, wood, chemical, oil and food.

However, these separation devices could optimize the cost-benefit ratio by manipulating their performance parameters, thus improving the technical conditions required in their design and subsequent manufacture, which can be achieved with a Good understanding of the dynamics of flow within. Despite its apparent simplicity, the dynamics of flow in a cyclone is complex, ${ }^{2}$ it includes features such as velocities, pressures, vorticity and in some cases the presence of several annular areas of reverse flow, for which the confined vortex flow theories do not predict satisfactorily observed phenomena. ${ }^{3}$ On the other hand, the problem associated with the detailed mathematical modeling of the flow profiles involves the solution of strongly coupled nonlinear partial differential equations - momentum and mass conservation, whose complete analytical solution is not yet known, however, Solve with a discretization method if an appropriate tool is available for the numerical solution. Computational Fluid Dynamics (CFD) simulation models provide an economical means to understand the complex flow dynamics within these equipment and how they are affected by changes in the original design or operating conditions, ${ }^{4}$ These calculations can be used over a wide range of flows, reducing the need for experimental tests, allowing predictions to be made in the design process and in the evaluation of industrial processes, reducing factors such as costs, risks and time, ${ }^{5}$ Thus providing a basis for decision making leading to the design of better performing systems.

The solution methodology for CFD models is to subdivide the domain into a large number of control volumes and convert the partial differential equations by integration on these control volumes into their algebraic equivalents. ${ }^{6}$

The result is a set of simultaneous algebraic equations that can be solved using iterative methods to obtain the field of dependent variable distributions relative to boundary conditions that define the specific problem, such as velocities and pressures.

This work simulates and evaluates the operation of one of these proposed geometries with the help of FLUENT ${ }^{\circledR}$ specialized software, which solves systems of partial differential equations using the discretization method of finite volumes, and allows to observe the behavior of the gas in the Cyclone interior, when comparing the gas phase tangential velocity profiles of a cyclone with geometry and flow conditions given by ${ }^{7}$ and compare these results with experimental data.

\section{Numerical analysis}

The equations applied for the numerical analysis in this study are the mass conservation and Navier-Stokes averaged Reynolds equations (RANS), to solve these equations the CFD code for the solution of the computational model was used.

The mass conservation and Navier-Stokes averaged Reynolds (RANS) equations in three dimensions are solved under the following assumptions, steady-state, Newtonian fluid, turbulent, incompressible, and three-dimensional flow. 
Continuity equation

$$
\frac{\partial}{\partial x_{i}}\left(\begin{array}{c}
-------- \\
\rho u_{i}+\rho^{\prime} u_{i}^{\prime}
\end{array}\right)=0
$$

Momentum equation

$$
\frac{\partial}{\partial x_{i}}\left(\rho u_{i}^{----} u_{j}\right)=\rho g_{i}-\frac{\partial p}{\partial x_{j}}+\frac{\partial\left(\tau_{i j}\right)_{e f f}}{\partial x_{i}}+S_{h}
$$

\section{Turbulence models}

Turbulent flows are characterized by having fields of fluctuating velocities. These fluctuations in turn cause constant changes in transported quantities such as amount of movement, energy, concentration of species, among others. This phenomenon of small scale and high frequency has a high cost to simulate computationally so it is necessary to make some simplifications to reduce the calculation time.

\section{Standard k-epsilon model}

It is a semi-empirical model based on the transport equations for turbulent energy $(\mathrm{k})$ and for the dissipation of turbulent epsilon energy $(\varepsilon)$. In the derivation of the model, it is assumed that the flow is totally turbulent and that the effects of molecular viscosity are negligible. Therefore, the $\mathrm{k}-\varepsilon$ model is only valid for fully turbulent flows.

Turbulent kinetic energy (k)

$$
\begin{aligned}
& \frac{\partial(\rho \bar{u} \kappa)}{\partial x}+\frac{\partial(\rho \bar{v} \kappa)}{\partial y}=\frac{\partial}{\partial x}\left[\left(\mu+\frac{u_{t}}{\sigma_{k}}\right) \frac{\partial k}{\partial x}\right]+ \\
& \frac{\partial}{\partial y}\left[\left(\mu+\frac{u_{k}}{\sigma_{k}}\right) \frac{\partial k}{\partial y}\right]+P_{k}+G_{k}-\rho \varepsilon-Y_{M}
\end{aligned}
$$

Dissipation of turbulent kinetic energy $(\varepsilon)$

$$
\begin{aligned}
& \frac{\partial(\rho \bar{u} \varepsilon)}{\partial x}+\frac{\partial(\rho \bar{v} \varepsilon)}{\partial y}=\frac{\partial}{\partial x}\left[\left(u+\frac{u_{t}}{\sigma_{\varepsilon}}\right) \frac{\partial \varepsilon}{\partial x}\right]+ \\
& \frac{\partial}{\partial y}\left[\left(u+\frac{u_{\varepsilon}}{\sigma_{\varepsilon}}\right) \frac{\partial \varepsilon}{\partial y}\right]+C_{1 \varepsilon} \frac{\varepsilon}{\kappa}\left[P_{\kappa}+C_{3 \varepsilon} G_{\kappa}\right]-C_{2 \varepsilon} \rho \frac{\varepsilon^{2}}{\kappa} \\
& P_{\kappa}=-\rho \mu_{t}\left[2\left(\frac{\partial \bar{u}}{\partial x}\right)^{2}+2\left(\frac{\partial \bar{v}}{\partial y}\right)^{2}+\left(\frac{\partial \bar{u}}{\partial y}+\frac{\partial \bar{v}}{\partial x}\right)^{2}\right] \\
& G_{\kappa}=-\beta g_{i} \frac{\mu_{t}}{\sigma_{\theta}} \frac{\partial \bar{T}}{\partial y} \\
& \mu_{t}=\rho C_{\mu} \frac{\kappa^{2}}{\varepsilon} \\
& Y_{M}=2 \rho \varepsilon M_{t}^{2} \\
& M_{t}=\sqrt{\frac{\kappa}{a^{2}}}
\end{aligned}
$$

Where $a$ is the speed of sound

$$
a=\sqrt{\gamma R T}
$$

\section{RNG k-epsilon model}

It is similar to the Standard k-epsilon model but includes some modifications

i. The RNG model has an additional term in the equation of $(\varepsilon)$.

ii. The effect of eddies on turbulence is included in this model.

iii. The RNG theory improves the analytical formula of Prandtl numbers while the standard model uses constants specified by the user.

iv. While the standard model operates in the fully developed turbulence flow region, The RNG theory consists of an analytically derived differential formula that takes into account the effects of the region where low numbers of turbulent Reynolds predominate. The effective use of this, however, depends on the treatment given to the mesh near the wall.

\section{Realizable k-epsilon model}

The term realizable means that the model satisfies certain limitations in the term of the normal efforts consistently with the physics of the turbulent flows, to understand it we must consider that the Boussinesq approximation and the definition of the turbulent viscosity are combined to obtain the following expression for the normal Reynolds stress in an incompressible flow,

$$
\overline{u_{i}^{\prime} u_{i}^{\prime}}=\frac{2}{3} \kappa-2 v_{t} \frac{\partial u}{\partial x}
$$

The realizable $\kappa-\varepsilon$ model differs from traditional k-epsilon models by the following

a. A new equation for turbulent viscosity involving the variable $\mathrm{C} \mu$, Originally proposed by Reynolds.

b. A new equation for the dissipation of turbulent kinetic energy. Based on the mean root equation of the vorticity fluctuation.

Using the equation of turbulent viscosity equation 7 , to obtain a relation for the turbulent kinematic viscosity given by

$$
v_{t} \equiv \frac{\mu_{t}}{\rho}
$$

You have to do the normal stress $\overline{u_{i}^{\prime} u_{i}^{\prime}}$ which is by definition a positive amount, becomes negative, therefore "non-realizable" when the following is true

$$
\frac{\kappa}{\varepsilon} \frac{\partial \bar{u}}{\partial x}>\frac{1}{3 C_{\mu}} \approx 3.7
$$

The Realizable $\kappa-\varepsilon$ model was proposed by Shit et al. In 1995, to address deficiencies of the traditional $\kappa-\varepsilon$ models used, adopting a new equation for turbulent viscosity involving the variable $C$ Originally proposed by Reynolds as mentioned above and an equation for the dissipation $(\varepsilon)$ based on the dynamic equation gives the vorticity fluctuation, therefore, the equations for the realizable $\kappa-\varepsilon$ model is, 
Turbulent kinetic energy $(\mathrm{k})$

$$
\begin{aligned}
& \frac{\partial(\rho \bar{u} \kappa)}{\partial x}+\frac{\partial(\rho \bar{u} \kappa)}{\partial y}=\frac{\partial}{\partial x}\left[\left(\mu+\frac{u_{t}}{\sigma_{k}}\right) \frac{\partial k}{\partial x}\right]+ \\
& \frac{\partial}{\partial y}\left[\left(\mu+\frac{u_{k}}{\sigma_{k}}\right) \frac{\partial k}{\partial y}\right]+P_{k}+G_{k}-\rho_{\varepsilon}-Y_{M}
\end{aligned}
$$

Disipación de la energía cinética turbulenta $(\varepsilon)$

$$
\begin{aligned}
& \frac{\partial(\rho \bar{u} \varepsilon)}{\partial x}+\frac{\partial(\rho \bar{v} \varepsilon)}{\partial y}=\frac{\partial}{\partial x}\left[\left(\mu+\frac{u_{t}}{\sigma_{\varepsilon}}\right) \frac{\partial \varepsilon}{\partial x}\right]+ \\
& \frac{\partial}{\partial y}\left[\left(\mu+\frac{u_{\varepsilon}}{\sigma_{\varepsilon}}\right) \frac{\partial \varepsilon}{\partial y}\right]+\rho C_{1}-C_{2 \varepsilon} \rho \frac{\varepsilon^{2}}{\kappa+\sqrt{v \varepsilon}}+ \\
& C_{1 \varepsilon} \frac{\varepsilon}{\kappa} C_{3 \varepsilon} G_{b}
\end{aligned}
$$

Where we have to

$$
C_{1}=\operatorname{Max}\left[0.43, \frac{\eta}{\eta+5}\right], \eta=S \frac{\kappa}{\varepsilon}, S=\sqrt{2 S_{i j} S_{i j}}
$$

The difference between the $\kappa-\varepsilon$ model and the realizable $\kappa-\varepsilon$ model is that $C_{\mu}$ it is not constant, its form is now

$$
C \mu=\frac{1}{\left(4.04+\sqrt{6} \cos \varphi \frac{\kappa U^{*}}{\varepsilon}\right)}
$$

Where

$$
\begin{aligned}
& U^{*}=\sqrt{S_{i j} S_{i j}+\Omega_{i j} \Omega_{i j}} \\
& \Omega_{i j}=\overline{\Omega_{i j}}-\varepsilon_{i j k} w_{k} \\
& \varphi=\frac{1}{3} \cos ^{-1}(\sqrt{6 W}) \\
& W=\frac{S_{i j} S_{j k} S_{k i}}{\bar{S}} \\
& \bar{S}=\sqrt{S_{i j} S_{i j}} \\
& S_{i j}=\frac{1}{2}\left(\frac{\partial \overline{u_{j}}}{\partial x_{i}}+\frac{\partial \overline{u_{i}}}{\partial x_{j}}\right)
\end{aligned}
$$

\section{Spalart allamaras model}

It is a model of an equation that uses the Boussinesq theory to model the transport equation of turbulent kinetic energy. This model was designed specifically for aerospace applications and is gaining popularity in turbo machinery applications.

$$
\begin{aligned}
& \frac{\partial(\rho \overline{\bar{v}})}{\partial t}+\frac{\partial\left(\rho \bar{v} u_{i}\right)}{\partial x_{i}}= \\
& G_{v}+\frac{1}{\sigma_{v}^{-}}\left[\frac{\partial}{\partial x_{j}}(\mu+\rho \bar{v}) \frac{\partial \bar{v}}{\partial x_{j}}+C b_{2} \rho\left(\frac{\partial \bar{v}}{\partial x_{j}}\right)^{2}\right]-Y v+S_{-}
\end{aligned}
$$

$$
\begin{aligned}
& \mu_{t}=\rho \bar{v} f_{v 1} \\
& f_{v 1}=\frac{\chi^{3}}{\chi^{3}+C_{v 1}^{3}} \\
& \chi \equiv \frac{\bar{v}}{v} \\
& G_{v}=C_{b 1} \rho \tilde{S}_{v}^{-} \\
& \tilde{S}=S+\frac{\tilde{v}}{\kappa^{2} d^{2}} f_{v 2} \\
& f_{v 2}=1-\frac{\chi}{1+\chi f_{v 1}} \\
& S=\sqrt{2 \Omega_{i j} \Omega_{i j}}, \Omega_{i j}=\frac{1}{2}\left(\frac{\partial u_{i}}{\partial x_{j}}-\frac{\partial u_{j}}{\partial x_{i}}\right]^{-1 / 6} \\
& Y v=C_{w 1} \rho f_{w}\left(\frac{\tilde{v}}{d}\right)^{2}, f_{w}=g\left[\frac{1+C_{w 3}^{6}}{g^{6}+C_{w 3}^{6}}\right]^{\frac{1}{v}} \\
& g=r+C_{w 2}\left(r^{6}-r\right), r \equiv \frac{\tilde{S}^{2} d^{2}}{{ }^{2}}
\end{aligned}
$$

Constant Models

$$
\begin{aligned}
& C_{b 1}=0.1335, C_{b 2}=0.622, \sigma_{\tilde{v}}=\frac{2}{3}, C_{v 1}=7.1 \\
& C_{w 1}=\frac{C_{b 1}}{\kappa^{2}}+\frac{\left(1+C_{b 2}\right)}{\sigma_{\tilde{v}}}, C_{w 2}=0.3, C_{w 3}=2.0 \\
& \kappa=0.4187
\end{aligned}
$$

\section{Case study}

For the study we used a Stairmand cyclone model of high efficiency Table 1, whose geometric and operating data, were taken from the open literature of, ${ }^{7}$ having the following dimensions Figure 1. As the working fluid, air with an inlet flow rate of $0.08 \mathrm{~m}^{3} \mathrm{~s}^{-1}$ at a viscosity of $1.7894 \mathrm{E}-05 \mathrm{~kg} \mathrm{~m}^{-1} \mathrm{~s}^{-1}$ and density of $1.225 \mathrm{~kg} \mathrm{~m}^{-3}$.

Table I Dimensions of the cyclone separator

\begin{tabular}{lllllll}
\hline $\mathbf{a} / \mathbf{D c}$ & b/Dc & De/Dc & S/Dc & h/Dc & H/Dc & B/Dc \\
\hline 0.5 & 0.2 & 0.5 & 0.5 & 1.5 & 4 & 0.36 \\
\hline
\end{tabular}

\section{Simulation conditions}

Numerically the FLUENT ${ }^{\circledR}$ commercial code in its version 6.3 was used, using the finite volume method to discretize the transport equations. The SIMPLE (Semi-Implicit Method for Pressure-Linked Equations) algorithm was used for the pressure-velocity coupling.

This segregated method allows solving the Navier-Stokes equations starting from a presumed pressure to obtain preliminary velocity values, which will be used to find a new pressure, and so on 
until convergence. A second order scheme was also used to interpolate the variables on the surface of the control volume.

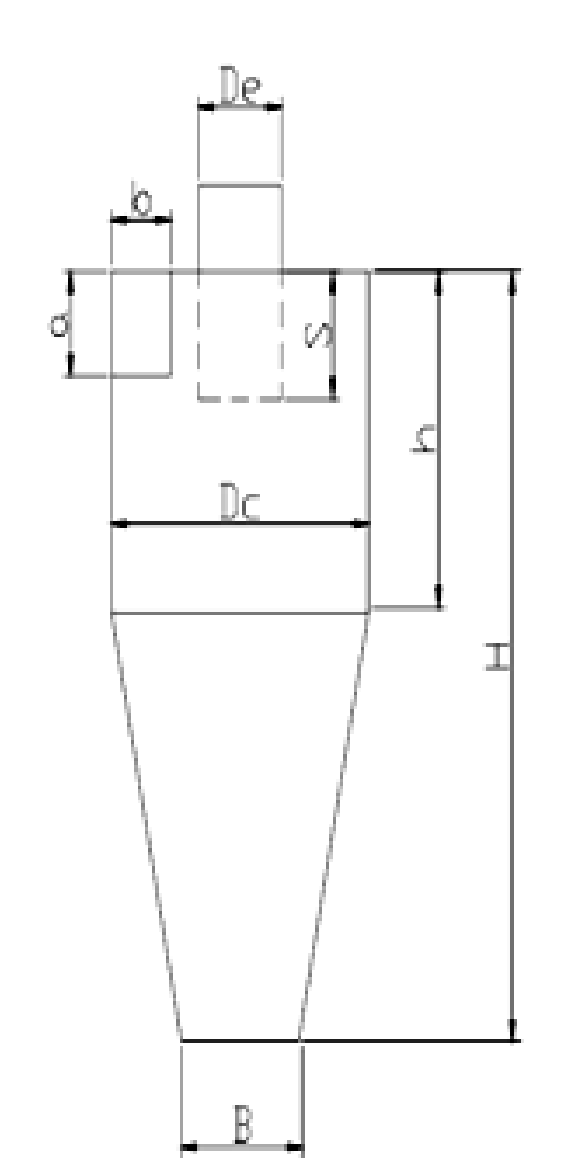

Figure I Dimensions of the cyclone separator.

The simulations were carried out on an IBM Works Station computer with an Intel (R), dual-core Xeon (R) IV, $3.0 \mathrm{GHz}$ processor, 8 GB RAM, CPU $5160 @ 3.00$ GHz, in which They required approximately 600 iterations to reach convergence in each model.

\section{Validation}

The validation is obtained by comparing the tangential velocity profiles obtained at different heights 32,38 and $41 \mathrm{~cm}$ below the vortex finger, using different turbulence models, Standard k-epsilon, RNG k- epsilon, Realizable k-epsilon And Spalart Allamaras, we then compared these tangential velocity profiles with the experimental data obtained with Laser Anemometry Dopler ${ }^{7}$ to the same heights, these experimental measures will serve to contrast the results of the simulation. ${ }^{8}$

\section{Results and discussion}

For the study to be performed tangential velocity profiles were considered within the separator, at different levels 32,38 and $41 \mathrm{~cm}$, Figure 2, using different turbulence models.

To perform the meshing, the GAMBIT ${ }^{\circledR}$ preprocessor was used. By means of a previous evaluation of the effect of the mesh refinement, a nodal network with tetrahedral elements was finally selected for the simulation. In Figure 3, the structure for the high efficiency Stairmand cyclones, which has 25881 nodes respectively, is schematically illustrated to ensure a reasonable prediction of the speed profiles within the device. ${ }^{9}$

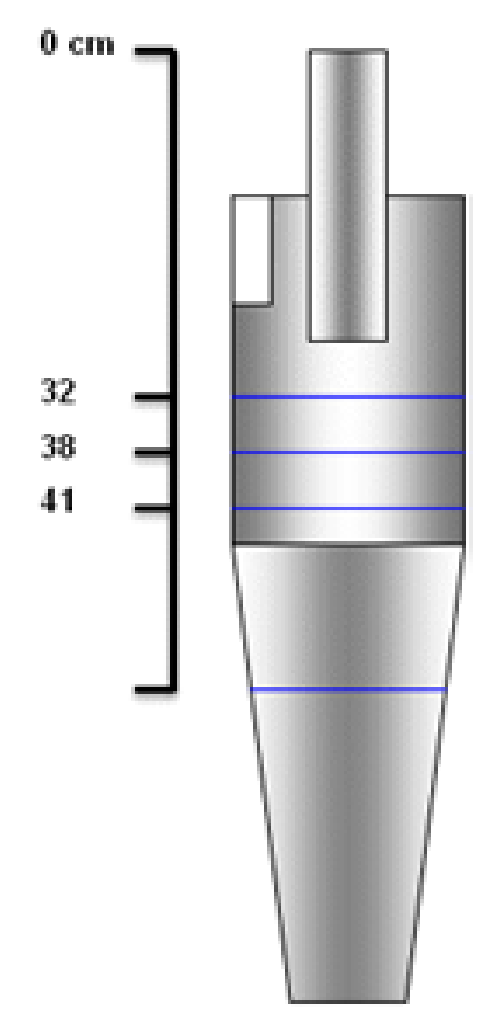

Figure 2 Lines at different heights for the calculation of tangential velocities.

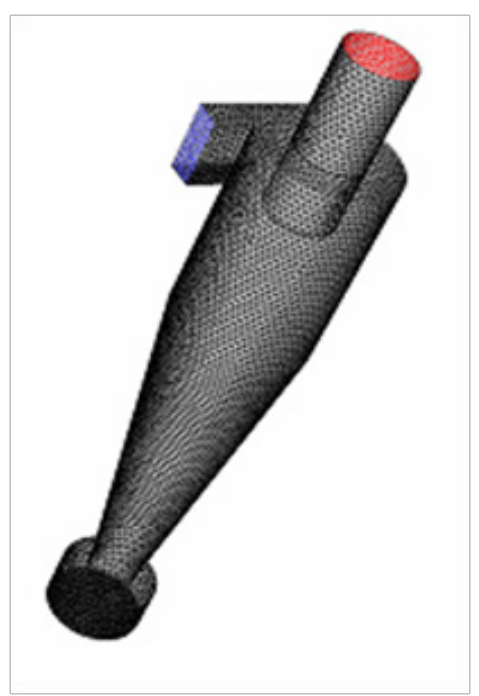

Figure 3 Cyclone mesh.

Para realizar el mallado, se empleó el preprocesador $G A M B I T^{\circledR}$. Mediante una evaluación previa del efecto del refinamiento de la malla, se seleccionó finalmente una red nodal con elementos tetraédricos para la simulación. En la Figure 3, se ilustra esquemáticamente la estructura para el ciclones Stairmand alta eficiencia, el cual cuenta con 25881 nodos respectivamente, para garantizar una predicción razonable de los perfiles de velocidad en el interior del dispositivo. ${ }^{10}$ 
After performing the simulations the profiles of the tangential velocity are obtained at different heights, and are compared with the experimental tangential velocity profiles (Figure 4-6 \& Table 2-4).

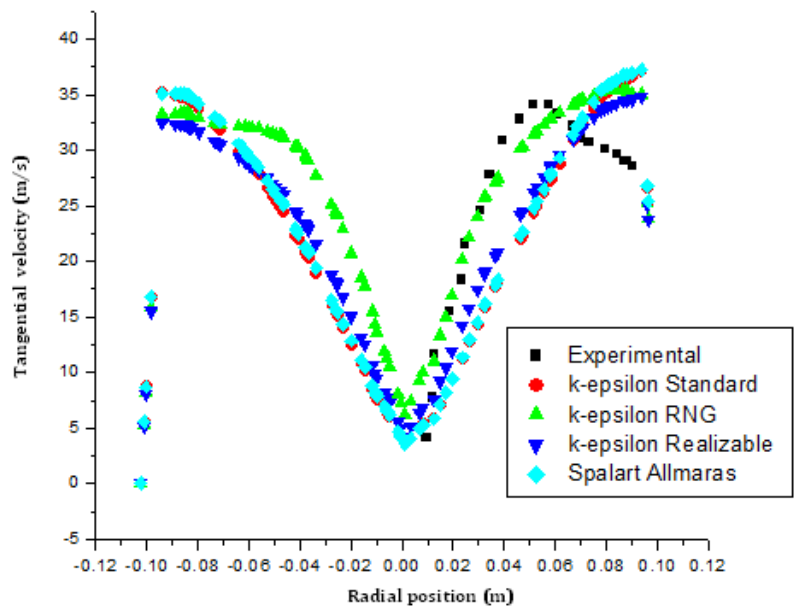

Figure 4 Tangential velocity at $32 \mathrm{~cm}$ below the vortex finder.

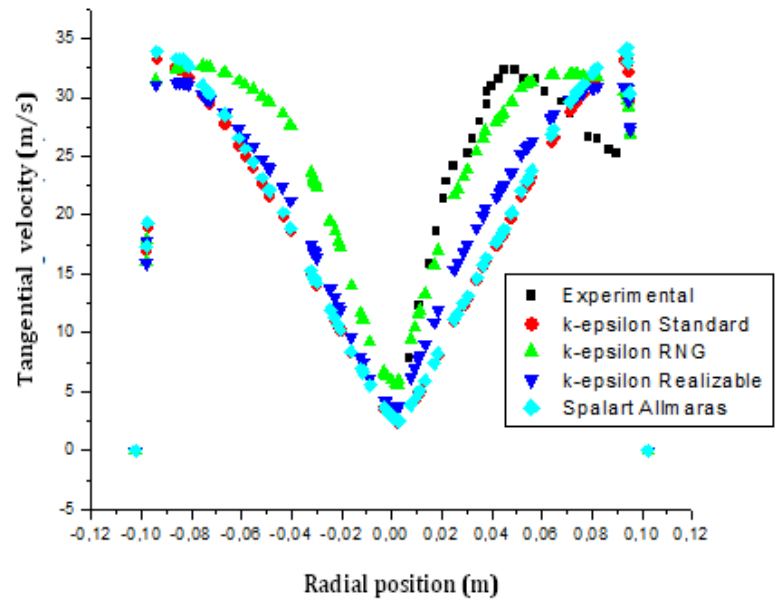

Figure 5 Tangential velocity at $38 \mathrm{~cm}$ below the vortex finder.

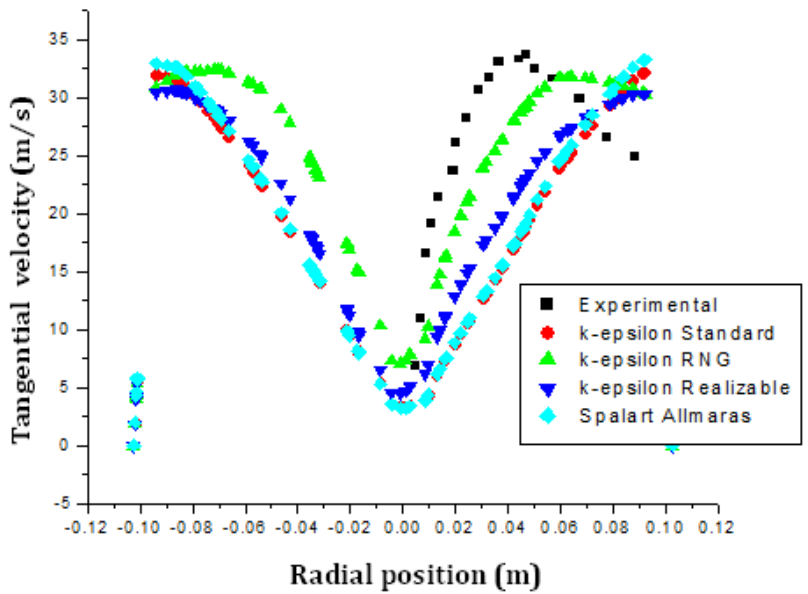

Figure 6 Tangential velocity at $4 \mathrm{I} \mathrm{cm}$ below the vortex finder.
Table 2 Relative error for different turbulence models at $32 \mathrm{~cm}$ below the vortex finder

\begin{tabular}{ll}
\hline Turbulence model & Relative error (\%) \\
\hline Standard k-epsilon & 3.62 \\
RNG k-epsilon & 2.38 \\
Realizable k-epsilon & 4.81 \\
Spalart Allmaras & 8.36
\end{tabular}

Table 3 Relative error for different turbulence models at $38 \mathrm{~cm}$ below the vortex finder

\begin{tabular}{ll}
\hline Turbulence model & Relative error (\%) \\
\hline Standard k-epsilon & 4.12 \\
RNG k-epsilon & 2.42 \\
Realizable k-epsilon & 5.21 \\
Spalart Allmaras & 8.49 \\
\hline
\end{tabular}

Table 4 Relative error for different turbulence models at $4 \mathrm{I} \mathrm{cm}$ below the vortex finder

\begin{tabular}{ll}
\hline Turbulence model & Relative error (\%) \\
\hline Standard k-epsilon & 5.38 \\
RNG k-epsilon & 3.72 \\
Realizable k-epsilon & 5.42 \\
Spalart Allmaras & 8.52 \\
\hline
\end{tabular}

As can be seen in Figures 4-6 the tangential velocity profiles present a trend or behavior similar to the experimental data, with a better approximation of the k-epsilon RNG turbulence model with respect to all the models studied since Presents a relative error of $2.32 \%$ for $32 \mathrm{~cm}$ below the vortex finder, $2.42 \%$ for $38 \mathrm{~cm}$ and $3.72 \%$ for $41 \mathrm{~cm}$, measured with respect to the experimental data, these values being smaller compared with the other models of turbulence studied, the behavior of the gas inside the separator Can be seen in Figure 7, where the velocity changes from the wall to the center of the cyclone as well as the velocity changes in the vicinities of the vortex seeker due to the current flow of the double spiral that starts at the apex of the cone. ${ }^{11}$

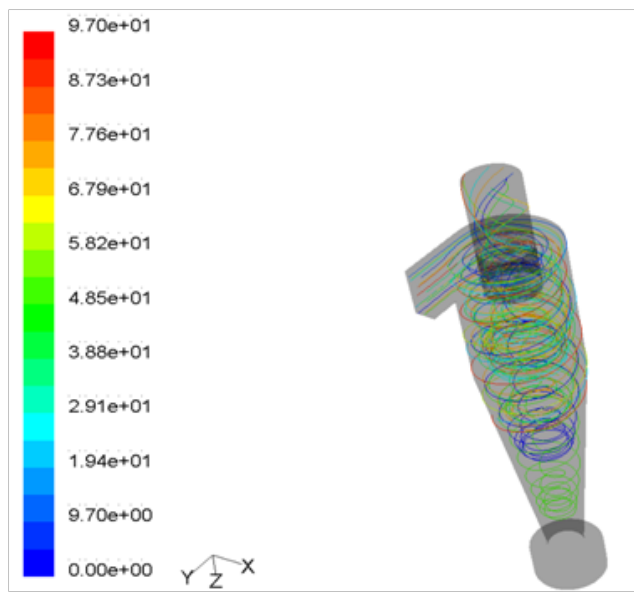

Figure 7 Pathline of air inside the cyclonic separator for RNG k-epsilon model. 
As the turbulence model closest to the experimental data is the RNG k-epsilon (Figures 8) (Figures 9) show the tangential and axial velocity contours respectively for this turbulence model.

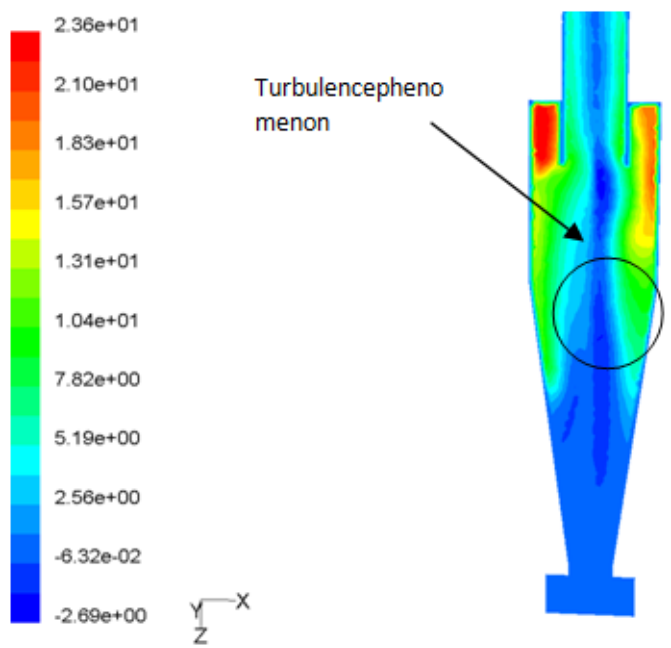

Figure 8 Tangential velocity contour for RNG k-epsilon model.

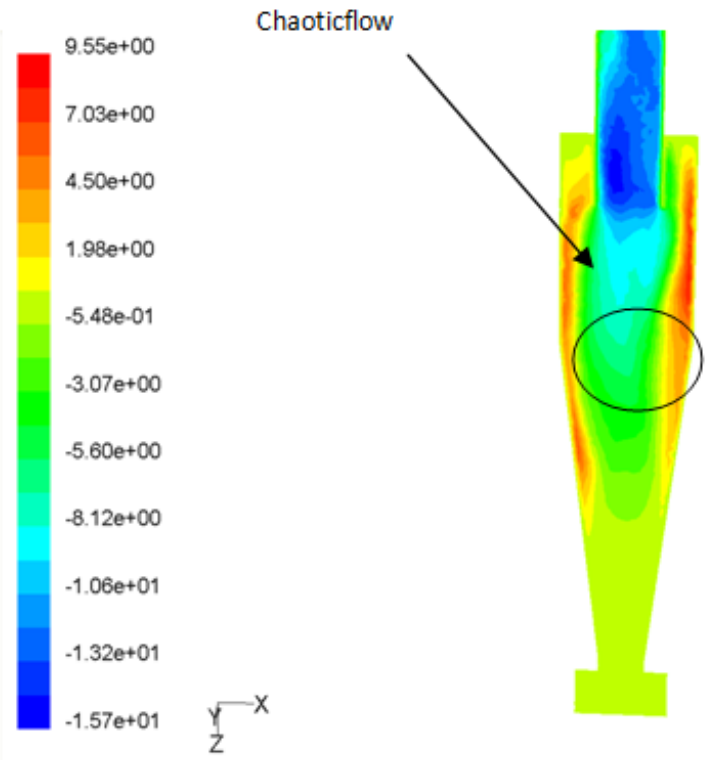

Figure 9 Axial velocity contour for RNG k-epsilon model.

Figure 8 , for the tangential velocity contour in an axial plane of the separator, the presence of oscillations in the vortex finder is observed. This is due to the unstable structure the vortex, which contributes to the entrainment of particles in the gas outlet (Carry-over), which directly affects the separation efficiency of the cyclone. ${ }^{12}$

In Figure 9, the axial velocity contour shows that the flow of current entering the cyclone collides with the stream that is directed to the vortex finder generating a chaotic flow in the outskirts of the localizer, consequently the velocity of the gas decreases strongly and Is redirected in the opposite direction, which influences the energy loss and the pressure drop of the cyclone

\section{Conclusion}

Different turbulence models were used to model the behavior of the gas inside the separating cyclone, namely, Standard k-epsilon, RNG k-epsilon, Realizable k-epsilon and Spalart Allamaras, we compared the profiles of tangential velocities, with experimental, geometric and operational data, ${ }^{7}$ as well as the study of the behavior of the gas in single phase inside the cyclonic separator, observing the axial and tangential velocity contours, in an axial plane of the separator for the model that is closer to the experimental calculation RNG k-epsilon.

The results of this simulation performed with CFD in cyclones at steady state are quite acceptable, because the behavior of the turbulence models used present very approximate results to the experimental values and their use will depend on what they want to look for in the study Of the efficiency of separation, therefore it is always necessary to carry out different investigations in this sense.

The finite volume method applied to fluid dynamics problems constitutes a useful and important tool for the conceptualization of the phenomenon to be studied since its formulation, establishing a relationship between the approach schemes used and the physical effects involved in the transport phenomena analyzed.

\section{Acknowledgements}

We thank the referees who have provided a number of comments and suggestions that have improved the content and quality of the manuscript.

\section{Conflict of interest}

The authors declare no conflict of interest.

\section{References}

1. Koch W, Lich W. New desing approach boosts cyclone efficiency. Chemical Engineering. 1977;84:241-249.

2. Witt PJ, Mitón LJ, Wu Jy Shepherd IC. Validation of CFD model for Predicting gas flow in a Cyclone. CHEMICA99. Australia, 1999. p. 246-251.

3. Gimbum J, Chuah TG, Fakhul Razi A. The influence of temperature and inlet velocity on cyclone pressure drop:a CFD study. Chemical Engineering and Processing. 2005;44(1):7-12.

4. Garcia Daniel, AlzateHader, Quintana Germán. Simulación del patrón de flujo en fase simple para diferentes diseños de separadores ciclónicos. Revista de Investigaciones Aplicadas. 2009;3(2):11-20.

5. Ranade V. Computational flow modeling for Chemicals reactor engineering. USA: Academic Press; 2001.

6. Pantakar, Suhas. Nuemrical heat transfer and fluid flow. Computational and Physical Processes in Mechanics and Thermal Sciences. Taylor \& Francis, USA, 1980. 197 p.

7. Slack MD, Prasad RO, Bakker A, et al. Advances in cyclone modelling using unstructured grids. IChemE. 2000;78(8):1098-1104.

8. Bernardo, Peres AP, Mori M. Computational study of cyclone flow fluid dynamics using a different inlet section angle. Thermal Engineering. 2005;4(1):18-23.

9. Cortés Cristobal, Gil Antonia. Modelling the gas and particle flow inside cyclone separators. Progress in Energy and Combustion Science. 2007;33(5):409-452. 
10. Zambrano H, A Brito, JG Márquez. Applications of Computational Fluid Dynamics to Study: Slurry Flow in pipeline for Heavy and Extraheavy Oil. SPE. 2014;9 p.

11. Zambrano H, Bencomo A. Numerical simulation of a gas stirred ladle, Selected Topics of Computational and Experimental Fluid Mechanics. Springer. 2015. p. 271-280.
12. Zambrano H, Sigalotti L, Franklin Peña Polo, et al. Turbulent models of oil flow in a circular pipe with sudden enlargement. Applied Mathematical Modelling. 2015;39(21):6711-6724. 